\title{
ADN circulant : une source d'information pour la détection et le suivi du cancer
}

On sait depuis plus de 50 ans que le plasma sanguin contient de petites quantités d'ADN libre circulant, de l'ordre d'une dizaine de nanogrammes par millilitre, cette concentration pouvant s'élever significativement, jusqu'à 0,5 microgramme par millilitre, chez les patients atteints de cancer. Plusieurs études ont montré qu'on pouvait, à partir de cet $\mathrm{ADN}$ circulant, détecter des modifications génétiques spécifiques des processus cancéreux comme des mutations de l'oncogène K-ras (dans les cancers du pancréas, colorectaux et pulmonaires) et du gène suppresseur de tumeur $p 53$ (dans les cancers pulmonaires, du sein et les hépatocarcinomes), une hyperméthylation du gène $p 16 / C D K N 2 A$ (dans les cancers pulmonaires et les hépatocarcinomes) et des altérations des microsatellites (cancers pulmonaires, colorectaux, de la tête et cou). La prévalence de ces altérations génétiques de l'ADN circulant varie cependant, selon les maladies et les gènes étudiés, de $13 \%$ (mutations de $p 53$ dans les cancers du sein) à $80 \%$ (mutations de $K$-ras dans les tumeurs pancréatiques) (pour revue, voir [1]). Notre groupe a récemment montré que l'étude de l'ADN circulant permet de détecter les altérations génétiques associées au carcinome hépatocellulaire (CHC) [2], un des cancers les plus fréquents de l'Afrique subsaharienne, du fait de l'association de deux facteurs de risque très communs: l'infection chronique par le virus de l'hépatite B (VHB) $(\mathrm{m} / \mathrm{s}$ $1990, n^{\circ} 3, p$. 306) et l'intoxication alimentaire par l'aflatoxine B1, une mycotoxine qui contamine une grande partie des sources d'alimentation traditionnelles. Cette mycotoxine est un carcinogène hépatique extrêmement puissant et induit l'appari-

sur le codon 249 du gène $p 53$ (AGG vers AGT, substitution d'une arginine par une sérine). Si ces deux facteurs de risque de CHC sont associés, la mutation est présente dans $70 \%$ des tumeurs hépatiques [3].

Depuis une douzaine d'années, le Centre International de Recherche sur le Cancer (CIRC) évalue, en Gambie, l'efficacité de la vaccination contre le VHB dans la prévention du CHC. En effet, dans ce pays, près de $20 \%$ de la population est porteuse chronique du VHB, et des adduits protéiques de l'aflatoxine sont détectables dans le sérum de près de $95 \%$ des individus. Nous avons utilisé les collections de sérum constituées par le CIRC pour évaluer la prévalence de la mutation du codon 249 du gène p53, identifiée par la présence d'un polymorphisme de restriction après amplification par PCR de l'exon 7 de ce gène. Nos résultats montrent la présence de cette mutation sur l'ADN circulant de $36 \%$ des patients atteints de CHC, $15 \%$ de ceux souffrant d'une cirrhose hépatique et $6 \%$ des individus sains, porteurs ou non du VHB. En revanche, aucun patient européen, atteint de CHC, n'est porteur de cette mutation [3].

Ainsi, en Gambie, l'étude de l'ADN circulant chez les patients atteints de CHC permet de détecter la mutation du gène codant pour p53, la corrélation avec sa présence sur l'ADN tumoral étant estimée à plus de $60 \%$. On peut envisager que la présence de cette mutation, chez les patients souffrant de cirrhose hépatique, permette une détection précoce du CHC. Cette hypothèse est en cours d'évaluation dans le cadre d'une étude prospective. Dans la population exposée à l'aflatoxine, la mutation de $p 53$ est observée dans les cellules hépatiques normales, ce qui suggère que sa présence est aussi un marqueur molécu- laire d'exposition à l'aflatoxine, et non pas de détection précoce de cancer. Dès lors, l'étude de l'ADN circulant pourrait avoir un impact pratique non seulement pour la détection ou le suivi des patients atteints de $\mathrm{CHC}$, mais aussi dans le cadre d'études épidémiologiques destinées à identifier des individus à risque au sein d'une population.

On ne connaît pas l'origine et la nature exacte de l'ADN circulant mais la détection de mutations somatiques spécifiques indique qu'il pourrait provenir des cellules tumorales. Sa présence dans le sérum de patients atteints de cancer pourrait être la conséquence d'une circulation de cellules tumorales témoignant d'un processus micro-métastatique, ou d'un relargage passif d'ADN tumoral secondaire à la mort de cellules cancéreuses in situ. Une hypothèse plus audacieuse serait celle d'un processus actif de sortie de l'ADN des cellules [1]. L'étude de ces processus revêt à présent une importance majeure, et il est bien possible que nous ne soyons pas au bout de nos surprises.

1. Anker P, Mulcahy H, Chen XQ, Stroun M. Detection of circulating tumour DNA in the blood (plasma/serum) of cancer patients. Cancer Metastasis Rev 1999; 18: 65-73.

2. Kirk GD, Camus-Randon AM, Mendy M, Goedert JJ., Hainaut P, Montesano R. 249ser p53 mutations in plasma from hepatocellular carcinoma, cirrhotic, and healthy patients from The Gambia, West Africa. J Natl Cancer Inst 2000; 92 : 148-53.

3. Montesano R, Hainaut P, Wild C. Hepatocellular carcinoma: from gene to public health. J Natl Cancer Inst 1997; 89 : 1844-51.

\section{Pierre Hainaut}

Centre International de recherche sur le Cancer, Groupe de cancérogenèse moléculaire, 150, cours Albert-Thomas, 69372 Lyon, France. 